\title{
Gastric Cancer: Biological Markers as Prognostic Factor
}

\begin{abstract}
Layane Barreto Costa ${ }^{1}$, Lucas Solon Dias de Farias ${ }^{1}$, Noele Gurgel d'Ávila ${ }^{1}$, Irami Araújo-Neto ${ }^{1}$, Francisco Irochima Pinheiro ${ }^{2}$, Eduardo Pereira de Azevedo² ${ }^{2}$ Amália Cinthia Meneses do Rêgo ${ }^{2}$, Fausto Pierdoná Guzen ${ }^{2}$ and Irami Araúio-Filho ${ }^{3}$
\end{abstract}

${ }^{1}$ Potiguar University, Rio Grande do Norte, Brazil

${ }^{2}$ Department in Biotechnology, Potiguar University, Brazil

${ }^{3}$ Department of Surgery, Potiguar University Brazil

Submission: November 28, 2017; Published: December 11, 2017

*Correspondence Address: Irami Araújo Filho, Professor of the Department of Surgery and Titular Chief of the Laboratory of Simulation in the Teaching of Health of the Potiguar University, Brazil, Tel: +55 84 988760206; Email: irami.filho@uol.com.br

Abstract

The present study had as objective to conduct a literature review on the gastric adenocarcinoma and the influence of biological markers as prognostic factors and mortality, contributing to future forms of treatment. A review of the literature on the subject, including the latest research on the topic, published in the last two years. The studies selected were divided into 11 categories:

i. E-cadherin in patients with gastric cancer;

ii. Expression of Her-2 gene;

iii.Integrin $\alpha v \beta 6$ and Metalloproteinase 9;

iv. Expression of MACC1 and KAI1 on metastasis and prognosis;

v. Value of CIP2A expression in advanced gastric cancer;

vi. Antigen MAGE-A and NY-ESO-1;

vii. $\quad$ CA 74-2, CA 125 and radical resection;

viii. $\quad$ AEG-1 Oncogene as prognostic biomarker;

ix. Associated with Macrophages tumors (TAM);

x. Cancer resectable Score;

xi. Natural therapy.

It was observed that gastric adenocarcinoma is currently the third global cause of death related to cancer. In Brazil, the stomach cancer is a third and fifth place of the cause of death for men and women, respectively. Studies have shown numerous biological markers, such as E-cadherin, TAM, CA 125, Metalloproteinase-9, which had a decisive impact on disease resections, bringing in a positive way the prognosis of the patients. In conclusion, one can say that the identification and determination of biological markers are of extreme importance in the treatment and prognostic evaluation of patients affected by gastric adenocarcinoma.

Keywords: Stomach neoplasms; Tumor biomarkers; Therapy; Prognostic factors; Survival; Mortality

\section{Introduction}

Gastric adenocarcinoma is a malignant neoplasm arising from gastric epithelium with a predominance in the region-den, which has multiple pyloric causal factors and requires environmental and genetic disorders to your development. Its evolution differs from symptomatic to asymptomatic frames, which contributes to late diagnosis, accelerated the progression of the dis ease, worsening of prognosis and high mortality rate. Patients who develop metastatic gastric cancer have a survival rate of less than a year with current therapies [1].

Stomach cancer is the third leading cause of cancer-related death in 2012, with deaths in the world 723,000. Is estimated as the fifth most common malignancy worldwide and is the third 
most frequent cause of death in males and females, a ratio of 2:1. In certain countries, is the most prevalent malignancy and the leading cause of death by cancer [2]. In Brazil, occupies the third place as a cause of cancer in men and fifth in women, except non-melanoma skin cancer. Currently, the gastric cancer is still a great burden on the resources and health units [3].

However, it was seen a reduction in your focus on rich countries like USA and England, being the 14th most prevalent type of cancer in the country. Noteworthy, the high rate of diagnoses in countries such as Japan, Russia, Chile and Costa Rica, justified by genetic factors [4]. The host bacterial virulence genes and environmental factors contribute to the process of oncogenesis. The development of cancer is a complex process that involves many genes and steps, including the expression and regulation of multiple oncogenes and tumor suppressor genes [5]. Since the development of molecular biology and genetics, many tumor genes have been studied as relevant to the diagnosis, treatment, and prognosis of gastric cancer, including Ras, c-myc, Rb and E-cadherin [2]. Resides in the respect the importance of studying and knowing the genes implicated in gastric carcinogenesis as a prognostic factor, because it influences the development of more targeted and specific therapies for this cancer, raising the survival and reducing mortality [6].

Therefore, this review aimed to search the most recent discoveries in relation to tumor suppressor genes, oncogenes involved and receptors related to gastric cancer in addition to observing the Association of the presence of these oncogenes with survival rates, prognosis and mortality of patients affected by adenocarcinoma of the stomach.

\section{Materials and Methods}

This work was done from an electronic search in the databases PubMed, Scopus and Embase search portal. We collected data from systematic review, randomized clinical trials, cohort studies and literary reviews, using the key words: stomach neoplasms; tumor biomarkers; therapy; prognostic factors; survival; mortality. The method presented the following guiding question: "what are the main results and scientific evidence identified in national and international bibliographical production of the last 2 years pertaining to stomach neoplasms, tumor biomarkers, therapy, prognostic factors, survival and mortality?" In the initial survey, the articles went through the evaluation of researchers (authors), in accordance with the following inclusion criteria: articles published in Portuguese, English or Spanish, to submit the combinations of the keywords selected, with publication date between 2015 and 2017 that were accessible. After the initial selection of material, were deleted the articles repeated in different databases and they focus on the gastric cancer and tumor biomarkers. Although picked by articles that cover effective updates in the treatment, the therapeutic failure was not used as a criterion for deletion, considering the particularity of the manifestations of each case.
In this context, the articles were read, selected and grouped into 11 categories:

i. E-cadherin in patients with gastric cancer; expression of Her-2;

ii. Integrin $\alpha v \beta 6$ and Metalloproteinase 9;

iii. KAI1 MACC1 expression and in the metastasis and prognosis;

iv. Meaning of CIP2A expression in advanced gastric cancer;

v. Antigens MAGE-A and NY-ESO-1;

vi. CA 74-2, CA 125 and radical resection;

vii. AEG-1 Oncogene as prognostic biomarker;

viii. Associated with Macrophages tumors (TAM);

ix. Cancer resectable Score;

x. Natural therapy.

\section{Discussion}

The objective of this study was to discuss the findings in the literature about the genes involved in the pathogenesis of gastric cancer and your impact on therapy, survival, and mortality in the population affected by this disease.

\section{E-cadherin in patients with Gastric cancer}

According to Rashid H et al. [1] Cadherin-1 gene (CDH1) is critical for maintenance of cell polarity and cell adhesion of epithelial tissue architecture and your expression is often reduced or lost in epithelial tumors, resulting in invasion and metastasis. The hypermethylation gene CDH1 is crucial to reducing your expression, but the pattern of methylation differs according to ethnicity, and may be by different environmental exposures of the ethnic groups [2]. This study used 80 tissues of gastric cancer and adjacent normal tissues containing areas in Department of General Surgery and Minimally Invasive the Sher-i-Kashmir Institute of Medical Sciences (SKIMS) [1].

Was found in the promoter region of the gene CDH1 for being hypermethylated in $65 \%(52 / 80)$ of samples of gastric cancer. These 52.8 (15.38\%) showed methylation in both tissues (cancerous and normal) and $44(84.6 \%)$ cases showed methylation only in the area of the tumor [3]. Also, it has been seen that patients with metastatic lymph nodes affected have a bigger hypermethylation compared with patients without lymph nodes affected, moreover, was also increased the methylation in women than men and in larger tumors [1].

\section{Expression of HER-2}

The epidermal growth factor receptor 2 (HER-2)-human belongs to the family of the epidermal growth factor receptor encoded by a human gene located on the long arm of chromosome 17. Retrospective studies have reported that the positivity 


\section{Cancer Therapy \& Oncology International Journal}

of HER-2 is a prognostic factor associated with increased risk of local invasion and metastasis [4-6].

Meng et al. [4,7] studied the epidermal growth factor receptor human-2 (HER-2), which is an oncoprotein belonging to the family of transmembrane human epidermal growth factors. Your activity on tyrosine kinase plays important roles in proliferation, differentiation, migration and survival cell [4,7]. Originally, the HER-2 was widely studied in breast cancer and your typical expression was correlated with more aggressive tumor activity and a worse prognosis. Target successfully for trastuzumab for the treatment of breast cancer, studies of the HER-2 in other solid tumors were also analyzed. The HER-2 expression was detected in lung cancer, endometrial carcinoma of type I and esophageal cancer [5,6]. In gastric cancer, it was observed that the high expression of HER-2 is associated with aggression and adverse outcomes7.

The study by Chong et al. [8] with 103 cases assessed the proportion of gastric cancers positive for the epidermal growth factor receptor 2 (HER-2) human, because these patients may benefit from targeted therapy. Of the 103 cases, 14 cases were positive for HER-2 (13.6\%), being significantly higher in elderly patients and that showed extensive disease. Takahashi et al. [9] showed that the expression of HER2 in tumor tissue was observed in 6 to $23 \%$ of the cases of advanced gastric cancer.

\section{Trastuzumab therapy in HER-2 Positive Gastric Cancer}

Meng et al. stated that the study of trastuzumab for gastric cancer (ToGA) showed that the addition of trastuzumab chemotherapy was beneficial for advanced gastric cancer HER-2 positive. The trastuzumab was approved for the treatment of patients with metastatic HER-2 positive adenocarcinoma of the stomach or gastroesophageal junction [4]. Takahashi et al. [9] showed that trastuzumab is a humanized monoclonal antibody that induces antibody-dependent cellular cytotoxicity, inhibits HER-2-mediated signaling and prevents the cleavage of extracellular domain of HER-2. Following this line of research, Chong VH et al. [8], analyzed the ToGA test that evaluated patients with gastroesophageal adenocarcinoma 3,803 advanced untreated and 594 patients with Her-2 positive tumors randomized to receive chemotherapy (cisplatin/fluoropyrimidine) associated with trastuzumab and chemotherapy alone. The primary objective of raising the overall survival was achieved (13.8 months for chemotherapy combined and 11.1 months for isolated $/ \mathrm{p}=$ 0.0046). The trastuzumab so it was considered safe to be given as standard chemotherapy, the patient Her-2 positive [9-11].

\section{Av $\beta 6$ Integrin and Metalloproteinase 9}

Analyzing other markers, Lian et al. [12] reported that the $\alpha v \beta 6$ Integrin is a member of the family and is expressed only in epithelial cells, fibronectin (FN) as your primary binder. The expression of $\alpha v \beta 6$ is rare and can hardly be detected in normal epithelial cells, but has substantially high levels in response to injury and/or inflammation in epithelial tumors [12]. Previous studies demonstrated that the expression of $\alpha v \beta 6$ Integrin is involved in pathogenic processes of gastrointestinal malignancies, including cell adhesion, proliferation, apoptosis and secretion of matrix metalloproteinase. The MMP-9 (Metalloproteinases 9) were involved in the invasion and metastasis of tumors that are characterized by a zinc atom at the active site and classified according to homology in sequence and substrate affinity. The expression of $\alpha \mathrm{v} \beta 6$ and MMP-9 is closely correlated and can serve as a more efficient and effective prognostic index in patients with gastric cancer [13-15].

Among the 126 patients analyzed in this study, $34.92 \%$ were positive for av $\beta 6$ and $42.06 \%$ expression to expression of MMP9. The expression of av $\beta 6$ was associated with the Lauren's rank, TNM/N differentiation. While that MMP-9 was associated with stage TNM/T differentiation [16]. Survival analysis by the Kaplan-Meier curve showed that patients with expression of $\alpha v \beta 6$ or MMP-9 isolated died sooner than those with negative expression and that patients who were both $\alpha v \beta 6$ and MMP-9 positives obtained a shorter overall survival than those with opposite pattern [12]. In this same study, it was observed a significant mortality risk stratification when were evaluated four different combinations of levels of $\alpha \mathrm{v} \beta 6$ and MMP-9 (i.e. positive markers, negative markers, positive with MMP- $9 \alpha v \beta 6$ negative and negative $\alpha v \beta 6$ with MMP-9 positive) by your relative effect on survival. Kaplan-Meier curves showed that patients who were both $\alpha v \beta 6$ and MMP-9 positive died earlier $(26.45 \pm 5.54)$ than the other three groups $(5.90 \pm 53.40,51.54 \pm 6.86$ and $68.86 \pm$ 4.87, respectively, $\mathrm{p}=0.000$ ) [12].

The Cox model indicated that the positive expression of $\alpha v \beta 6$ and MMP-9, Lauren's rank diffuse, as well as high levels of $\mathrm{N}, \mathrm{M}$ and TNM were predictors of a poor prognosis in univariate analysis [11]. The biggest difference in survival rate was found among patients with both positive markers and those with the opposite pattern (both negative markers). Clinical follow-up data were obtained sufficient of all 126 patients, allowing the assessment of the association between protein change and outcome prognosis. Of these, $69(54.8 \%)$ cases were confirmed as cancer-related deaths in five years [12].

\section{KAI1 and MACC1 expression in the Metastasis and Prognosis}

The KAI1 is a tumor suppressor gene that acts by inhibiting phosphorylation of tyrosine B-catenin and stabilizing the complex E-cadherin-B-catenin to suppress tumor metastasis. In addition, inhibits the process mediated by B-catenin to prevent tumor angiogenesis and lymphangiogenesis [13-16]. The expression decreased or lost is linked to metastasis and prognosis of tumors as larynx, prostate carcinoma, breast carcinoma, lung carcinoma, gastric carcinoma, colon carcinoma and hepatocellular carcinoma. MACC1 is already connected to the promoter gene of Epithelial Mesenchymal Transition (MET), promoting the proliferation, invasion and metastasis of cancerous cells. It is still a factor Predictor of metastasis and prognosis for other types of 


\section{Cancer Therapy \& Oncology International Journal}

cancer (lung, liver, pancreatic, ovarian, gastric, malignant glioma, breast and cervical carcinoma) [11].

Lu et al. [16] analyzed tissue 325 of Gastric Adenocarcinoma (AG) by immunohistochemistry and as a result that the expression of MACC1 (metastasis associated with colon cancer 1) was significantly higher in tissues with AG than in control tissues, being positively correlated with tumor size, grade, invasiveness and advanced TNM staging. KAI1 expression (Kangai1) has been correlated with carcinogenesis, cancer metastasis and bad prognosis. The KAI1 is a tumor suppressor gene, so patients with positive expression of KAI1 had a significant increase in survival compared to negative KAI1. The metastasis and recurrence are the most common reasons of deaths in AG. The TNM staging is used, however, do not provide us with necessary information on the biological behavior of cancer and, why the need to search for biomarkers to predict recurrence and metastasis [17-19].

The overall survival time in patients with positive expression of MACC/AG and has been reduced from 56.1 months to 32.7 months. The survival time for patients who expressed KAI1 rose from 35.4 months to 52.6 months, compared to patients who did not have the suppressor gene expression in question [16].

\section{Meaning of CIP2A expression in advanced Gastric Can- cer}

Chen et al. [20] stated that the marker CIP2A (Cancerous Inhibitor of Protein Phosphatase 2A) is expressed in a variety of cancers. In this study was evaluated the expression and clinical significance of CIP2A in patients with advanced gastric cancer. CIP2A protein was expressed in 25 of 37 cancer tissue specimens. There was no correlation between the expression CIP2A and PGP, GST- $\pi$, Top-II and LRP. The expression of CIP2A may not have a prospective value to optimize the chemotherapy treatment regimens, but it can be an indicator for the prognosis of the patient. Uncontrolled cell proliferation is a characteristic of cancer cells and tumorigenesis is related to disordered expression of some key factors that participate in the regulation of cell cycle progression, differentiation, senescence and apoptosis [21]. Thus, the aberrant expression of some proteins may result in the formation of cancer in humans. For example, the inhibitor protein phosphatase 2A carcinogen (CIP2A) is a recently identified human oncoproteína that inhibits the degradation of c-MYC protein in cancer cells, highly expressed in different human cancers [22].

The MYC protein is a transcription factor all-in-one that has been associated with a wide range of cellular functions, such as cell cycle regulation, proliferation, growth, differentiation and metabolism. The MYC signalling abnormal was observed in human cancers and demonstrated that this factor promotes cell transformation and tumor progression [23]. Many studies have reported that phosphatase protein (PP2A) causes proteolytic degradation of oncoproteína, MYC, and prevents the malignant cells grow [24]. However, CIP2A can stabilize the MYC protein inhibiting PP2A activity and promotes the formation of tumor in vivo. To sum up, the survival rate of patients with positive CIP2A expression was significantly different from the negative patients [25]. It was observed that the CIP2A is expressed at low levels in most tissues not malignant, but is elevated in malignant cells by stabilizing the MYC protein by inhibition of PP2A activity and thus promotes tumor formation in vivo. CIP2A protein is expressed mainly in the cytoplasm and nucleus of cells from gastric cancer [23-26].

\section{H. pylori infection and expression of CIP2A}

Infection by $H$. pylori has cancerous relationship in the development of gastric cancer. In addition, it was shown that patients with gastric cancer very young (30 years) are less likely to become infected with $H$. pylori and have less exposure to environmental toxins, suggesting that hereditary factors may be more important than the $H$. pylori infection in tumorigenesis $[27,28]$. Chen et al. [20] still correlated to H. pylori infection and expression of CIP2A. The H. pylori infection is today considered a risk factor for gastric cancer. The continuing colonization of $H$. pylori in the stomach leads to a high risk of peptic ulcers and gastric cancer. It was discovered that the positive rate of CIP2A was much more prominent in the Hp-positive group compared to the Hp-negative group $(\mathrm{P}=0.009)$, suggesting that the $H$. pylori infection correlates with excessive expression of c-MYC, inducing the tumorigenesis of gastric mucosa. Thus, CIP2A gene may play a role in $H$. pylori infection related to gastric carcinogenesis [20].

\section{Antigen MAGE-A and NY-ESO-1}

Kerkar et al. studied two antigens of testicular cancer, esophageal squamous cell carcinoma in New York-1 (NY-ESO-1) and the family of the antigens of melanoma (MAGE), which represent promising immunotherapy targets due to the low expression of these antigens in not malignant tissue. Was found a significantly higher expression of MAGE-A ( $>50 \%$ in tumor cells) compared to NY-ESO-1 in various carcinomas [29]. Only two stained not carcinomas to MAGE-the thyroid follicular cancer and kidney cancer. In summary, MAGE-A is widely expressed in various typical histology of cancer prevalence and mortality. In addition, the statistical analysis showed that most of the cancers evaluated has an expression MAGE-significantly higher than the NY-ESO-1 [30].

Thus, Kerkar et al. [29] concluded that testicular cancer antigens represent immunotherapeutics ideal targets due to your restricted expression in normal tissue combined with high expression in malignantly transformed cells. MAGE-A is more widely expressed that NY-ESO-1 in a wide range of common carcinomas. Despite the classical vision of that, NY-ESO-1 is a promising target for immunotherapy; the study agrees that the NY-ESO-1 is not highly expressed in common carcinomas. The highest percentage of positivity of NY-ESO-1 is observed in gastric adenocarcinomas, present in seven patients of 50 cases of positive staining (14\%). 


\section{CA 72-4, CA 125 and Radical Resection}

Zhou et al. retrospectively reviewed all gastric cancer resections in patients undergoing surgical procedures 4671 and endoscopes carried out of between 2004 to 2014. The worst prognostic factors resulting from included high levels of CA72-4, CA 125 , positive resection margin and tumors in stage pIII-pIV [31]. The 5-year survival rate was significantly higher in patients with radical resection than those without this type of resection. Early detection of elevated serum levels of CA72-4, CA-125 and radical resection rather than palliative, may raise the rates of survival, especially for those with family history [31-33].

\section{AEG-1 oncogene as Prognostic Biomarker}

Luo $\mathrm{Y}$ et al. [34] studied the gene-1 astrocytic elevation (AEG-1), also known as metaderin (MTDH) that was first identified in 2002 as a new protein induced in astrocytes primary human infected by human immunodeficiency virus 1 (HIV) -1 and factor tumor necrosis factor $\alpha$ (TNF- $\alpha$ ) [34]. The AEG-1 gene is an oncogene, located on chromosome $8 \mathrm{q} 22$ region, and noted that the high expression your promoted proliferation, tumor progression or metastases in multiple carcinomas such as gastric cancer, neuroblastoma, breast cancer, prostate cancer and malignant glioma [35]. In addition, the AEG-1 can activate multiple molecular mechanisms to carry out its functions, including the nuclear factor $\kappa-\mathrm{B}(\mathrm{NF}-\kappa \mathrm{B})$, Phosphatidylinositol 3-kinase (PI3K) and c-Myc, Wnt/b-catenin by extracellular signal-regulated kinase (ERK), triggering protein 1 (AP-1) and No thyroid disease (NTLS). In addition, it was reported that AEG-1 elevates the expression of angiopoietina-1, matrix metalloproteinase-2 (MMP2), 1- $\alpha$ inducible factor by hypoxia (HIF- $\alpha$ ) and Tie2, which are essential in angiogenesis [36-38].

The evidence above shows that the AEG-1 is involved in the process of proliferation, infiltration and tumor metastasis. In addition, a meta-analysis to explore the relationship between the index of AEG-1 staining and clinical pathological features in squamous cell carcinoma (SCC), that provided clear information on the influence of AEG-1 in squamous cell carcinoma [37]. The analyses of subgroups have necessitated that the AEG-1 SI correlated significantly with all gastrointestinal cancers including squamous cell carcinoma (ESCC), esophagus colorectal carcinoma (CRC), gallbladder carcinoma (GBC), gastric carcinoma (GC), hepatocellular carcinoma (HCC) and pancreatic adenocarcinoma (PAC) [39].

According to the meta-analysis, the AEG-1 is a new biomarker that reflects the status of the aggression of the disease and prognostic value, which meant a new type of tumor marker to be studied. However, the results showed mostly the circumstances in Asia, once the population included in the meta-analysis consisted mainly of Asians. If the new biomarker is suitable for patients of other regions, it must be tested and evaluated in the upcoming clinical trials in different countries [35-37]. It was observed that the high rates of AEG-1 indicated effectively tu- mor progression aggravating the prognosis in gastrointestinal cancers. In addition, AEG-1 mediates resistance to medicines through multiple mechanisms34. Furthermore, it was reported that perifosin may be a targeted therapy drug that suppressed the gene expression AEG-1, inhibiting the Akt signaling pathway/GSK3b/C-MYC in GC [36]. In summary, the AEG-1 is a potential target to cure gastrointestinal cancer and clinical studies will be required in developing medicines AEG-1 inhibitors in order to explore therapeutic and prognostic value as your new biomarker tumor [38].

To conclude, the AEG-1 is actively involved in the process of tumor invasion, metastasis of lymph nodes and metastasis from a distance. In fact, AEG-1 plays a vital role in the process and appears to be an effective biomarker that reflects the status of prognosis of patients with gastrointestinal cancer $[39,40]$.

\section{Macrophages associated with Tumors (TAM)}

Kim et al. demonstrated that the tumor microenvironment plays a crucial role in many malignant tumors, and involves several factors, including immune cells, fibroblasts, blood vessels, extracellular matrix and soluble factors, among which, the macrophages are the immune most abundant populations [41]. The main functions and characteristics of macrophages associated with tumors (TAM) previously have been studied by many researchers. In general, the TAMs release numerous factors such as cytokines, chemokines and growth factors that influence the behaviors of the tumor cells [42]. It is considered that monocytes have a functional and phenotypic plasticity that allows them to differentiate into two states of polarization-macrophages M1 and M2-depending on the kind of tumor microenvironment in immune [43].

Macrophages induced by cytokines of the Th1 type (M1) as interferon- $\gamma$ and microbial stimuli, such as lipopolysaccharides, produce pro-inflammatory cytokines, chemokines and reactive nitrogen intermediates/oxygen. Thus, these cells are involved in antimicrobial activity and tumoricidal $[40,41]$. In contrast, alternatively activated macrophages (M2) are induced by Th2 cytokines, including interleukin-4 (IL-4), IL-10 and IL-13 and show immunoregulatory activity, anti-inflammatory and promoter of tumors [43].

In general, the TAMs are considered more alike with the phenotype M2 when compared to the M1. Therefore, the TAMs are associated with reduced survival of cancer patients promoting invasion, metastasis, angiogenesis and lymphangiogenesis. In fact, the TAMs were related to reduced survival of patients with many solid tumors (testicles, ovarian, melanoma, lung, endometrium, breast and kidney) [44]. However, several other studies in gastric carcinoma and colon-rectal cancers showed a better prognosis in patients with high density of TAM, which indicates that the functional role of these can be different depending on the type of fabric and, therefore, the type of cancer in which are enabled [41,42]. 


\section{Cancer Therapy \& Oncology International Journal}

\section{Cancer Resectable Score}

Qian et al. developed a prognostic scoring system simple and reliable for gastric cancer (GC) treated with the D2 lymphadenectomy is associated with gastrectomy combined with adjuvant chemotherapy [45]. A classification system of risk assessment of prognosis of three classes was established by integrating levels of hemoglobin, CEA in the serum, preoperative postoperative state of lymphovascular invasion (LVI) and lymph nodes (NRL). This system can identify the subsets of high-risk patients of stage II or III which will be forwarded for more intensive treatment, which has potential benefits of chemotherapy based on paclitaxel or oxaliplatin before chemotherapy administration adjuvant. Therefore, the scoring system with the three model classes is recommended to predict the prognosis [46]

Although the benefit of gastrectomy for patients with resectable GC is clear and that some kind of neoadjuvancy, perioperative chemotherapy or adjuvant therapy is necessary to improve the survival of patients, there is no international consensus on the best approach, resulting in different guidelines that vary between countries and regions [41-43]. One of the key findings of this study is that the current scoring system identified patients with different long-term forecasts inside every pTNM stage (I-III), suggesting a series of high-risk patients are underestimated using only the classification of pTNM. These high-risk subgroups eventually benefit from a more intensive postoperative treatment $[44,45]$.

To evaluate the role of prognostic score in several adjuvant chemotherapy regimens was also examined the difference in survival of chemotherapy of paclitaxel (Taxol)-, oxaliplatin and cisplatin by the model A and model B. the results showed patients who received paclitaxel showed better results, but only in the high-scoring group. No difference was observed in the group of low-scoring [39-42]. In model B, patients in the highrisk group also seemed to benefit from chemotherapy based on paclitaxel or oxaliplatin, but not in the low and intermediate risk groups. In addition, patients in low and high risk groups have not reached any survival benefit when subjected to cisplatin-based chemotherapy in models A and B [44-46]. The prognostic value of number of cytotoxic agents was also examined in the model A and model B. The triple chemotherapy correlated with a better prognosis compared to duplicate therapy or monotherapy, but again only in high-risk subgroups according to the models $\mathrm{A}$ and B [45-47].

\section{Natural Therapy}

In relation to natural treatments, Gao et al. [48] evaluated the herbs chinese medicines (CHM), in the treatment of stage IV gastric adenocarcinoma. The average survival was higher in patients who made use of the CHM to the detriment of those who did not, from 18 months to 9 months. Of the 294, 13 were correlated with favorable results, acting on some targets the proliferation of epidermal growth receptor, fibroblast growth factor, proliferating cell nuclear antigen and metastasis process cancer in the families of collagen, fibronectin 1 and matrix metalloproteinases [47-50].

\section{Conclusion}

The prognosis and mortality rate of gastric adenocarcinoma is closely related to the expression of tumor markers and study specific oncogenes in this review. That is, genes such as E-cadherin, HER- 2 receptor, the Integrin $\alpha v \beta 6$, MMP- 9 and CIP2A are directly involved in oncogênese of gastric cancer. The KAI1 and the MACC1, on the other hand, relate to the prognosis and severity of the disease, and therefore of important research.

The studies of tumors associated with macrophages (TAMs) has your importance as it draws attention of the role of the macrophages activated in tumor genesis by promoting the theory that inflammatory cytokines influence the behavior and growth of tumor cells. Already the AEG-1 is an important prognostic biomarker, as it brings to your positivity, an indication of possible metastatic involvement and lymph node invasion. Worth mentioning also the MAGE-A antigens and NY-ESO-1 immunotherapy in promising, for your high expression in tissues, including malignant carcinomas definitely acid. You can't forget the importance of radical resection of tumor lesion when the serum levels of CA 72-4 and CA 125 are elevated in patients with positive family history for gastric neoplasm, since it was considered a superior conduct to the palliative resection. In addition, finally, essential to recall the value diagnosis of $H$. pylori infection in the digestive epithelium, a significant role for attacker, mutagenic and predictor of potential gastric cancer lesion.

Therefore, the identification and determination of these receptors/markers in therapeutic and research wins predictive of disease, since it helps the clinical and pathophysiological understanding, supporting the alternative therapy to be employed.

\section{References}

1. Rashid H, Alam K, Afroze D, Yousuf A, Banday M, et al. (2016) Hypermethylation Status of E-Cadherin Gene in Gastric Cancer Patients in a High Incidence Area. Asian Pac J Cancer Prev 17(6): 2757-2760.

2. Zhong K, Chen W, Xiao N, Zhao J (2015) The clinicopathological significance and potential drug target of E-cadherin in NSCLC. Tumour Biol 36(8): 6139-6148.

3. Zeng W, Zhu J, Shan L, Han Z, Aerxiding P, et al. (2015) The clinicopathological significance of CDH1 in gastric cancer: a meta-analysis and systematic review. Drug Des Devel Ther 9: 2149-2157.

4. Meng X, Huang Z, Di J, Mu D, Wang Y, et al. (2015) Expression of Human Epidermal Growth Factor Receptor-2 in Resected Rectal Cancer. Medicine (Baltimore). 94(47): e2106.

5. Wang HB, Liao XF, Zhang J (2017) Clinicopathological factors associated with HER2-positive gastric cancer: A meta-analysis. Medicine (Baltimore) 96(44): e8437.

6. Lei YY, Huang JY, Zhao QR, Jiang N, Xu HM, et al. (2017) The clinicopathological parameters and prognostic significance of HER2 expression in gastric cancer patients: a meta-analysis of literature. World J Surg Oncol 15(1): 68 


\section{Cancer Therapy \& Oncology International Journal}

7. Galdy S, Lamarca A, McNamara MG, Hubner RA, Cella CA, et al. (2017) HER2/HER3 pathway in biliary tract malignancies; systematic review and meta-analysis: a potential therapeutic target? Cancer Metastasis Rev 36(1): 141-157.

8. Chong VH, Telisinghe PU, Tan J, Abdullah MS, Chong CF (2016) Profile of HER2 +ve Gastric Cancers in Brunei Darussalam. Asian Pac J Cancer Prev 17(5): 2555-2558

9. Takahashi N, Furuta K, Taniguchi H, Sasaki Y, Shoji H, et al. (2016) Serum level of hepatocyte growth factor is a novel marker of predicting the outcome and resistance to the treatment with trastuzumab in HER2-positive patients with metastatic gastric cancer. Oncotarget. 7(4): 4925-4938.

10. Matsusaka S, Nashimoto A, Nishikawa K, Miki A, Miwa H, et al. (2016) Clinicopathological factors associated with HER2 status in gastric cancer: results from a prospective multicenter observational cohort study in a Japanese population (JFMC44-1101). Gastric Cancer 19(3): 839851

11. Zhang X, Wu Y, Gong J, Lu Z, Zhou J, et al. (2014) Trastuzumab combined with chemotherapy in patients with HER2-positive chemo-refractory advanced gastric or gastro-esophageal junction adenocarcinoma] Zhonghua Zhong Liu Za Zhi 36(3): 223-227.

12. Lian PL, Liu Z, Yang GY, Zhao R, Zhang ZY, Chen YG, Zhuang ZN, Xu KS. Integrin $\alpha \mathrm{v} \beta 6$ and matrix metalloproteinase 9 correlate with survival in gastric cancer. World J Gastroenterol 22(14): 3852-3859.

13. Niu J, Li Z (2017) The roles of integrin $\alpha v \beta 6$ in cancer. Cancer Lett 403 : 128-137.

14. Liu Y, Wang Y, Teng Z, Chen J, Li Y, et al. (2017) Matrix metalloproteinase 9 expression and survival of patients with osteosarcoma: a meta-analysis. Eur J Cancer Care 26(1).

15. Gong L, Wu D, Zou J, Chen J, Chen L, et al. (2016) Prognostic impact of serum and tissue MMP-9 in non-small cell lung cancer: a systematic review and meta-analysis. Oncotarget 7(14): 18458-18468.

16. Lu G, Zhou L, Zhang X, Zhu B, Wu S, et al. (2016) The expression of metastasis-associated in colon cancer-1 and KAI1 in gastric adenocarcinoma and their clinical significance. World J Surg Oncol 14(1): 276.

17. Virgilio E, Proietti A, D’Urso R, Cardelli P, Giarnieri E, et al. (2017) Measuring Intragastric Tumor Markers in Gastric Cancer Patients: a Systematic Literature Review on Significance and Reliability. Anticancer Res 37(6): 2817-2821.

18. Richman DM, Tirumani SH, Hornick JL, Fuchs CS, Howard S, et al. (2017) Beyond gastric adenocarcinoma: Multimodality assessment of common and uncommon gastric neoplasms. Abdom Radiol (NY) 42(1): 124-140.

19. Wang G, Fu Z, Li D (2015) MACC1 overexpression and survival in solid tumors: a meta-analysis. Tumour Biol 36(2): 1055-1065.

20. Chen JS, Wu BB, Bao HL, Du JM, Zhang SC, et al. (2015) Relationship between CIP2A expression, and prognosis and MDR-related proteins in patients with advanced gastric cancer. Int J Clin Exp Pathol 8(11): 15007-15012.

21. Chen KF, Yen CC, Lin JK, Chen WS, Yang SH, et al. (2015) Cancerous inhibitor of protein phosphatase $2 \mathrm{~A}$ (CIP2A) is an independent prognostic marker in wild-type KRAS metastatic colorectal câncer after colorectal liver metastasectomy. BMC Cancer 15: 301

22. Chao TT, Maa HC, Wang CY, Pei D, Liang YJ, et al. (2016) CIP2A is a poor prognostic factor and can be a diagnostic marker in papillary thyroid carcinoma. APMIS 124(12): 1031-1037.

23. Routila J, Bilgen T, Saramäki O, Grénman R, Visakorpi T, et al. (2016) Copy number increase of oncoprotein CIP2A is associated with poor patient survival in human head and neck squamous cell carcinoma. J Oral Pathol Med 45(5): 329-337.
24. Khanna A, Pimanda JE (2016) Clinical significance of cancerous inhibitor of protein phosphatase 2A in human cancers. Int J Cancer 138(3): 525-532.

25. He XX, Ding L, Lin Y, Shu M, Wen JM, et al. (2015) Protein expression of HER2, 3, 4 in gastric cancer: correlation with clinical features and survival. J Clin Pathol 68(5): 374-380.

26. Cristóbal I, Zazo S, Torrejón B, Pedregal M, Madoz-Gúrpide J, et al. (2017) CIP2A confirms its prognostic value in triple-negative breast cancer. Oncogene 36(23): 3357-3358.

27. Kawanaka M, Watari J, Kamiya N, Yamasaki T, Kondo T, et al. (2016) Effects of Helicobacter pylori eradication on the development of metachronous gastric cancer after endoscopic treatment: analysis of molecular alterations by a randomised controlled trial. Br J Cancer 114(1): 21-29.

28. Oshizawa Y, Sugimoto M, Sato Y, Sahara S, Ichikawa H, et al. (2016) Factors associated with healing of artificial ulcer after endoscopic submucosal dissection with reference to Helicobacter pylori infection, CYP2C19 genotype, and tumor location: Multicenter randomized trial. Dig Endosc 28(2): 162-172.

29. Kerkar SP, Wang ZF, Lasota J, Park T, Patel K, et al. (2016) MAGE-A is More Highly Expressed Than NY-ESO-1 in a Systematic Immunohistochemical Analysis of 3668 Cases. J Immunother 39(4):181-187.

30. Park TS, Groh EM, Patel K, Kerkar SP, Lee CC, et al. (2016) Expression of MAGE-A and NY-ESO-1 in Primary and Metastatic Cancers. J Immunother 39(1): 1-7.

31.Zhou F, Shi J, Fang C, Zou X, Huang Q (2016) Gastric Carcinomas in Young (Younger than 40 Years) Chinese Patients: Clinicopathology, Family History, and Postresection Survival. Medicine (Baltimore) 95(9): e2873.

32. Wang Z, Xu J, Shi Z, Shen X, Luo T, et al. (2016) Clinicopathologic characteristics and prognostic of gastric cancer in young patients. Scand J Gastroenterol 51(9): 1043-1049.

33. Kim JH, Jun KH, Jung H, Park IS, Chin HM (2014) Prognostic Value of Preoperative Serum Levels of Five Tumor Markers (Carcinoembryonic Antigen, CA19-9, Alpha-fetoprotein, CA72-4, and CA125) in Gastric Cancer. Hepatogastroenterology. 61(131): 863-869.

34. Luo Y, Zhang X, Tan Z, Wu P, Xiang X, et al. (2015) Astrocyte Elevated Gene-1 as a Novel Clinicopathological and Prognostic Biomarker for Gastrointestinal Cancers: A Meta-Analysis with 2999 Patients. PLoS One 10(12): e0145659.

35. Fakhri B, Lim KH (2017) Molecular landscape and sub-classification of gastrointestinal cancers: a review of literature. J Gastrointest Oncol 8(3):379-386.

36. Petrelli F, Ghidini M, Barni S, Steccanella F, Sgroi G, et al. (2017) Prognostic Role of Primary Tumor Location in Non-Metastatic Gastric Cancer: A Systematic Review and Meta-Analysis of 50 Studies. Ann Surg Oncol 24(9): 2655-2668.

37. Ge H, He X, Guo L, Yang X (2017) Clinicopathological significance of HSP27 in gastric cancer: a meta-analysis. Onco Targets Ther 10: 45434551.

38. Ge H, Yan Y, Guo L, He X, Yang X (2017) Prognostic and clinical significance of VEGFR-3 in gastric cancer: A meta-analysis. Clin Chim Acta 474: $114-119$

39. Park KJ, Yu MO, Song NH, Kong DS, Park DH, et al. (2015) Expression of astrocyte elevated gene-1 (AEG-1) in human meningiomas and its roles in cell proliferation and survival. J Neurooncol 121(1): 31-39.

40. He W, He S, Wang Z, Shen H, Fang W, et al. (2015) Astrocyte elevated gene-1(AEG-1) induces epithelial-mesenchymal transition in lung cancer through activating Wnt/ $\beta$-catenin signaling. BMC Cancer 15: 107. 


\section{Cancer Therapy \& Oncology International Journal}

41. Kim KJ, Wen XY, Yang HK, Kim WH, Kang GH (2015) Prognostic Implication of M2 Macrophages Are Determined by the Proportional Balance of Tumor Associated Macrophages and Tumor Infiltrating Lymphocytes in Microsatellite-Unstable Gastric Carcinoma. PLoS One 10(12) e0144192.

42. Hu W, Li X, Zhang C, Yang Y, Jiang J, et al. (2016) Tumor-associated macrophages in cancers. Clin Transl Oncol 18(3): 251-258.

43. Yang L, Zhang Y (2017) Tumor-associated macrophages: from basic research to clinical application. J Hematol Oncol 10(1): 58.

44. Alves AM, Diel LF, Lamers ML (2017) Macrophages and prognosis of oral squamous cell carcinoma: A systematic review. J Oral Pathol Med.

45. Qian J, Qian Y, Wang J, Gu B, Pei D, et al. (2015) A clinical prognostic scoring system for resectable gastric cancer to predict survival and benefit from paclitaxel- or oxaliplatin-based adjuvant chemotherapy. Drug Des Devel Ther 10: 241-258.

46. Wang ZX, Yang XL, He MM, Wang F, Zhang DS, et al. (2016) The Efficacy of Adjuvant FOLFOX6 for Patients With Gastric Cancer after D2
Lymphadenectomy: A Propensity Score-matched Analysis. Medicine (Baltimore) 95(16): e3214.

47. Park SH, Sohn TS, Lee J, Lim DH, Hong ME, et al. (2015) Phase III Tria to Compare Adjuvant Chemotherapy With Capecitabine and Cisplatin Versus Concurrent Chemoradiotherapy in Gastric Cancer: Final Report of the Adjuvant Chemoradiotherapy in Stomach Tumors Trial, Including Survival and Subset Analyses. J Clin Oncol 33(28): 3130-3136.

48. Gao L, Hao J, Niu YY, Tian M, Yang X, et al. (2016) Network pharmacology dissection of multiscale mechanisms of herbal medicines in stage IV gastric adenocarcinoma treatment. Medicine (Baltimore) 95(35): e4389.

49. Wong VK, Law BY, Yao XJ, Chen X, Xu SW, et al. (2016) Advanced research technology for discovery of new effective compounds from Chinese herbal medicine and their molecular targets. Pharmacol Res 111: 546555 .

50. Jorgensen JT (2014) Role of human epidermal growth factor receptor 2 in gastric cancer: biological and pharmacological aspects. World J Gastroenterol 20(16): 4526-4535.

\section{Your next submission with Juniper Publishers will reach you the below assets}

- Quality Editorial service

- Swift Peer Review

- Reprints availability

- E-prints Service

- Manuscript Podcast for convenient understanding

- Global attainment for your research

- Manuscript accessibility in different formats

( Pdf, E-pub, Full Text, Audio)

- Unceasing customer service

Track the below URL for one-step submission https://juniperpublishers.com/online-submission.php 